\title{
Continuous-Time Markowitz's Problems in an Incomplete Market, with No-Shorting Portfolios
}

Hanqing $\operatorname{Jin}^{1}$ and Xun Yu Zhou ${ }^{2}$

${ }^{1}$ Department of Systems Engineering and Engineering Management, The Chinese University of Hong Kong, Shatin, Hong Kong, hqjin@se.cuhk.edu.hk

2 Department of Systems Engineering and Engineering Management, The Chinese University of Hong Kong, Shatin, Hong Kong, Tel.: 852-2609-8320, fax: 852-2603-5505, xyzhou@se.cuhk.edu.hk. Supported by the RGC Earmarked Grants CUHK 4175/03E, CUHK418605, and Croucher Senior Research Fellowship. We also thank an anonymous referee for helpful comments that have led to an improved version.

Dedicated to Professor Kiyosi Itô for His 90th Birthday

Summary. Continuous-time Markowitz's mean-variance portfolio selection problems with finite-time horizons are investigated in an arbitrage-free yet incomplete market. Models with unconstrained and no-shorting portfolios are tackled respectively. The sets of the terminal wealths that can be replicated by admissible portfolios are characterized in explicit terms. This enables one to transfer the original dynamic portfolio selection problems into ones of static, albeit constrained, optimization problems in terms of the terminal wealth. Solutions to the latter are obtained via certain dual (static) optimization problems. When all the market coefficients are deterministic processes, mean-variance efficient portfolios and frontiers are derived explicitly.

AMS 1991 subject classifications. 91B28, 60H10

Key words and phrases. Mean-variance portfolio selection, continuous time, arbitrage-free, incomplete market, Lagrange multiplier, backward stochastic differential equation, attainable wealth set, replication

\section{Introduction}

Markowitz's Nobel-prize-winning work on single-period mean-variance portfolio selection [23] has laid down the foundation for modern financial portfolio theory. Nevertheless, as pointed out in a recent survey paper [28] the 
mean-variance approach has received little attention in the context of dynamic investment planning, especially in the continuous time setting. Most continuous-time portfolio selection models in literature assume that the investor seeks to maximize expected utility, which is a departure from the meanvariance model. While the utility approach was theoretically justified by von Neumann and Morgenstern [25], in practice "few if any investors know their utility functions; nor do the functions which financial engineers and financial economists find analytically convenient necessarily represent a particular investor's attitude towards risk and return" [24].

Research on faithfully extending the Markowitz model to the dynamic setting has emerged in very recent years, starting with $\mathrm{Li}$ and $\mathrm{Ng}$ [17] where a discrete-time, multiperiod, mean-variance problem is solved explicitly using an embedding technique to cope with the non-applicability of dynamic programming caused by the variance term. Subsequently, in a series of papers $[30,20,18,2]$ various continuous-time Markowitz models have been investigated thoroughly with closed-form solutions obtained for most cases. Two main approaches are exploited to solve the problems. In the first approach, which is adopted in $[30,20,18]$, one transfers the underlying mean-variance problem into a family of indefinite stochastic linear-quadratic (LQ) optimal control problems, and then uses an elaborative completion-of-square technique, via one or more stochastic Riccati equations, to derive the solutions. This approach is inspired by the recent development in indefinite LQ control $[4,29]$, and is particularly effective when there is no constraint on the state variable (the wealth, that is). Since in this approach a mean-variance efficient portfolio is obtained dynamically and forwardly in the process of optimization, we call it a "forward approach" or "primal approach". In contrast, in the second approach which is taken in [2], an optimal terminal wealth is first identified by solving a static optimization problem, and then an efficient portfolio is obtained by replicating the optimal terminal wealth. This approach, which we call a "backward approach" or "dual approach", also widely known as that of equivalent (risk neutral) martingale measures, goes back to Harrison and Kreps [12] and Pliska [26]. It is particularly powerful in solving the Markowitz problem with additional wealth constraints, as demonstrated in [2].

In all the papers $[30,20,18,2]$, it is assumed that the dimension of the underlying Brownian motion, used to model the stock prices, is the same as the number of the stocks, and the covariance matrix has uniformly positive eigenvalues. This induces a complete market where the risk associated with any reasonable contingent claim can be completely hedged. Although in $[18,2]$ there are no-shorting or no-bankruptcy constraints, which essentially render the market incomplete, the aforementioned assumption is critical for the forward or backward approaches to work.

In this paper, we consider the continuous-time Markowitz problem in a market where the dimension of the Brownian motion is different from the number of the stocks, and all the market coefficients are random (i.e., the investment opportunity set is stochastic). In addition, we will attack the problem 
for two cases respectively: 1) portfolios are unconstrained; and 2) shorting is prohibited. (Strictly speaking, the unconstrained case can be regarded as a special case of the other. We treat it separately because it is simpler, and we intend to use it to first showcase the essential idea without having to involve too much technicality.) As discussed earlier none of the approaches taken in the previous related articles would work. To overcome, for each of the two cases, we will first characterize, in explicit terms, the so-called attainable terminal wealth set, namely the set of terminal wealths that can be replicated by admissible portfolios satisfying the respective constraint. Then we solve a static optimization problem on random variables of terminal wealth with the attainable terminal wealth set representing an additional constraint. The mean-variance efficient portfolios are then derived by replicating those optimal, attainable terminal wealths for both cases. In the general situation of random market coefficients, we will prove all the necessary existence and uniqueness results while suggesting a scheme of approaching the final solutions. This is then exemplified by the scenario of deterministic coefficients where we are able to solve the original problem in explicit and analytical forms.

While the present work attempts to tackle the continuous-time meanvariance portfolio selection in incomplete markets, there have been many works in literature devoted to continuous-time portfolio selection with incomplete markets, albeit in the realm of expected utility; see [5, 10, 11, 14, 15, 27] among others. It should be emphasized again that the existing results in the utility framework do not at all cover the mean-variance models for the main reason that the assumptions typically imposed on a utility function are not satisfied by a mean-variance model. To be specific, a typical utility function should satisfy several conditions, especially the one that its derivative must vanish at infinity; see, e.g., Karatzas and Shreve [15, p. 94, Definition 4.1]. (If proportional portfolios - portfolios being defined as proportions of wealth allocated to different stocks - are being considered, then the utility function must be further that its derivative is infinite at 0; see, e.g., [5].) These properties are crucial in deriving all the results with the expected utility and hence have been all along the standing assumptions in relevant literature. Unfortunately, the quadratic utility associated with the mean-variance model (if you must "embed" the mean-variance into the utility framework!) does not satisfy most of these assumptions. Consequently, one cannot apply a priori the results from the utility model. To the best of our knowledge the only preceding paper that deals with the Markowitz problem in an incomplete market is Lim [19] where a forward approach is applied together with a completion-of-market trick of [14] and nonlinear backward stochastic differential equation theory. However, only unconstrained portfolios are considered in [19]. In comparison, in the present paper we go with the backward approach, and solve constrained problems in incomplete markets.

The remainder of the paper is organized as follows. In section 2 we introduce the market under consideration along with some of its important proper- 
ties. Section 3 is devoted to some technical results on the pricing kernels that are vital for the subsequent analysis. Section 4 sets up the continuous-time Markowitz models. In sections 5-6 we respectively solve the two cases. Finally, section 7 concludes the paper.

\section{Market}

In this paper $T$ is a fixed terminal time and $\left(\Omega, \mathcal{F}, P,\left\{\mathcal{F}_{t}\right\}_{t \geq 0}\right)$ is a fixed filtered complete probability space on which is defined a standard $n$-dimensional Brownian motion $W(t) \equiv\left(W^{1}(t), \cdots, W^{n}(t)\right)^{\prime}$ with $W(0)=0$, and $\mathcal{F}_{t}=$ $\sigma\{W(s): 0 \leq s \leq t\}$ augmented by all $P$-null sets. We denote by $L_{\mathcal{F}}^{2}\left(0, T ; \mathbf{R}^{d}\right)$ the set of all $\mathbf{R}^{d}$-valued, $\mathcal{F}_{t}$-progressively measurable stochastic processes $f(\cdot)=\{f(t): 0 \leq t \leq T\}$ with $\|f(\cdot)\|_{L_{\mathcal{F}}^{2}\left(0, T ; \mathbf{R}^{d}\right)}:=\left(E \int_{0}^{T}|f(t)|^{2} d t\right)^{\frac{1}{2}}<$ $+\infty$, by $L_{\mathcal{F}}^{\infty}\left(0, T ; \mathbf{R}^{d}\right)$ the set of all $\mathbf{R}^{d}$-valued, essentially bounded, $\mathcal{F}_{t^{-}}$ progressively measurable stochastic processes $f(\cdot)$ with $\|f(\cdot)\|_{L_{\mathcal{F}}\left(0, T ; \mathbf{R}^{d}\right)}:=$ $\operatorname{esssup}_{(t, \omega) \in[0, T] \times \Omega}|f(t, \omega)|<+\infty$, and by $L_{\mathcal{F}_{T}}^{2}\left(\Omega ; \mathbf{R}^{d}\right)$ the set of all $\mathbf{R}^{d_{-}}$ valued, $\mathcal{F}_{T}$-measurable random variables $\eta$ such that $\|\eta\|_{L_{\mathcal{F}_{T}}^{2}\left(\Omega ; \mathbf{R}^{d}\right)}:=$ $\left(E|\eta|^{2}\right)^{\frac{1}{2}}<+\infty$. Throughout this paper, a $(t, \omega)$-null set is a null-set with respect to the product of the Lebesgue measure on $[0, T]$ and $P$ on $\Omega$, and a.s. signifies that the corresponding statement holds true with probability 1 (with respect to $P$ ).

Notation. We use the following additional notation:

$\mathbf{Q}^{d}$ : the set of $d$-dimensional vectors with rational components;

$\mathbf{R}_{+}^{d}$ : the set of $d$-dimensional vectors with nonnegative components;

$\mathbf{R}_{+}:=\mathbf{R}_{+}^{1}$;

$M^{\prime}$ : the transpose of any vector or matrix $M$;

$|M|:=\sqrt{\sum_{i, j} m_{i j}^{2}}$ for any matrix or vector $M=\left(m_{i j}\right)$;

$\alpha^{+}:=\max \{\alpha, 0\}$ for any real number $\alpha$;

$\alpha^{-}:=\max \{-\alpha, 0\}$ for any real number $\alpha$.

In the market under consideration in this paper, there are $m+1$ assets (or securities) being traded continuously. One of the assets is a bank account whose price process $S_{0}(t)$ is subject to the following differential equation:

$$
\left\{\begin{array}{l}
d S_{0}(t)=r(t) S_{0}(t) d t, \quad t \in[0, T] \\
S_{0}(0)=s_{0}>0
\end{array}\right.
$$

where the interest rate process $r(\cdot) \in L_{\mathcal{F}}^{\infty}(0, T ; \mathbf{R})$. Note that normally one would assume that $r(t) \geq 0$; yet this assumption is not necessary in our subsequent analysis. The other $m$ assets are stocks whose price processes $S_{i}(t)$, $i=1, \cdots, m$, satisfy the following stochastic differential equation (SDE):

$$
\left\{\begin{array}{l}
d S_{i}(t)=S_{i}(t)\left[\mu_{i}(t) d t+\sum_{j=1}^{n} \sigma_{i j}(t) d W^{j}(t)\right], t \in[0, T], \\
S_{i}(0)=s_{i}>0,
\end{array}\right.
$$


where $\mu_{i}(\cdot) \in L_{\mathcal{F}}^{\infty}(0, T ; \mathbf{R})$ and $\sigma_{i j}(\cdot) \in L_{\mathcal{F}}^{\infty}(0, T ; \mathbf{R})$ are the processes of appreciation and dispersion (or volatility) rates, respectively.

Denote

$$
\begin{aligned}
\sigma(t): & =\left(\sigma_{i j}(t)\right)_{m \times n}, \\
B(t) & \equiv\left(b_{1}(t), \cdots, b_{m}(t)\right)^{\prime}:=\left(\mu_{1}(t)-r(t), \cdots, \mu_{m}(t)-r(t)\right)^{\prime} .
\end{aligned}
$$

Consider an agent whose total wealth at time $t \geq 0$ is denoted by $x(t)$, and the dollar amount invested in stock $i, i=1, \cdots, m$, is $\pi_{i}(t)$. Assume that the trading of shares takes place continuously in a self-financing fashion (i.e., there is no consumption or income) and there are no transaction costs. Then the wealth process $x(\cdot)$ satisfies

$$
d x(t)=\left[r(t) x(t)+B(t)^{\prime} \pi(t)\right] d t+\pi(t)^{\prime} \sigma(t) d W(t), \quad x(0)=x,
$$

where $\pi(t)=\left(\pi_{1}(t), \cdots, \pi_{m}(t)\right)^{\prime}$ is the portfolio of the agent at time $t$, and $x$ is the initial wealth of the agent.

The following assumption will be imposed throughout this paper.

Basic Assumption (A): There exists $\theta \in L_{\mathcal{F}}^{\infty}\left(0, T, \mathbf{R}^{n}\right)$ such that $\sigma(t) \theta(t)=$ $B(t)$, a.s., a.e.t $\in[0, T]$.

The above assumption is satisfied if $\sigma(t)^{\prime} \sigma(t)$ is uniformly positive definite (i.e., there is $\delta>0$ such that $\sigma(t)^{\prime} \sigma(t) \geq \delta I_{n}$ a.s., a.e. $\in[0, T]$ ), in which case, necessarily, $n \leq m$, and there is a unique such $\theta$. In general, however, the process $\theta$, if it exists, may not be unique.

Definition 1. A portfolio (process) $\pi(\cdot)$ is said to be admissible if $\sigma(\cdot)^{\prime} \pi(\cdot) \in$ $L_{\mathcal{F}}^{2}\left(0, T ; \mathbf{R}^{n}\right)$. The set of all admissible portfolio is denoted by $\Pi$. A pair $(x(\cdot), \pi(\cdot))$ is called an (admissible) wealth-portfolio pair if $(x(\cdot), \pi(\cdot))$ satisfies (3).

Observe that under Assumption (A), for any $\pi(\cdot) \in \Pi, B(\cdot)^{\prime} \pi(\cdot)=$ $\theta(\cdot)^{\prime}\left[\sigma(\cdot)^{\prime} \pi(\cdot)\right] \in L_{\mathcal{F}}^{2}(0, T ; \mathbf{R})$. Hence by standard SDE theory a unique strong solution $x(\cdot) \equiv x^{\pi}(\cdot)$ exists for the wealth equation (3).

For any $\theta \in L_{\mathcal{F}}^{\infty}\left(0, T, \mathbf{R}^{n}\right)$, define

$$
H_{\theta}(t):=\exp \left\{-\int_{0}^{t}\left[r(s)+\frac{1}{2}|\theta(s)|^{2}\right] d s-\int_{0}^{t} \theta(s)^{\prime} d W(s)\right\} .
$$

Equivalently, $H_{\theta}(\cdot)$ can be defined as the unique solution to the following SDE

$$
\left\{\begin{array}{l}
d H_{\theta}(t)=-r(t) H_{\theta}(t) d t-H_{\theta}(t) \theta(t)^{\prime} d W(t), \\
H_{\theta}(0)=1
\end{array}\right.
$$

It is clear that for any $\theta \in L_{\mathcal{F}}^{\infty}\left(0, T, \mathbf{R}^{n}\right)$ there is a constant $c=c(\|$ $\left.\theta \|_{L_{\mathcal{F}}^{\infty}\left(0, T, \mathbf{R}^{n}\right)}\right)$ such that $E\left[\sup _{0 \leq t \leq T} H_{\theta}(t)^{2}\right] \leq c$. 
Define

$$
\Theta:=\left\{\theta \in L_{\mathcal{F}}^{\infty}\left(0, T, \mathbf{R}^{n}\right): \sigma(t) \theta(t)=B(t), \text { a.s., a.e. } t \in[0, T]\right\},
$$

and

$$
\hat{\Theta}:=\left\{\theta \in L_{\mathcal{F}}^{\infty}\left(0, T, \mathbf{R}^{n}\right): \sigma(t) \theta(t) \geq B(t) \text {, a.s., a.e.t } \in[0, T]\right\},
$$

where the greater or equal relation between two vectors is in the componentwise sense. It follows from Assumption (A) that $\emptyset \neq \Theta \subseteq \hat{\Theta}$.

Let $\theta \in \Theta$, and $(x(\cdot), u(\cdot))$ be an admissible wealth-portfolio pair. Then it is knows ([7, p. 22, Proposition 2.2]) that

$$
x(t)=H_{\theta}(t)^{-1} E\left(x(T) H_{\theta}(T) \mid \mathcal{F}_{t}\right), \text { a.s., } \forall t \in[0, T] .
$$

Definition 2. The market is said to be arbitrage-free if whenever a wealth process $x(\cdot)$ under an admissible portfolio satisfies $x(T) \geq 0$ a.s. and $P\{x(T)>$ $0\}>0$, it must hold that $x(0)>0$.

Arbitrage-free is a very weak market condition, for many optimization problems would become ill-posed in a non arbitrage-free market. It is easy to show that the market is arbitrage-free under Assumption (A). Conversely, if the market is arbitrage-free, then it can be proved, as in $[15$, p.12, Theorem 4.2], that there must be an $\mathcal{F}_{t}$-progressively measurable process $\theta$ satisfying $\sigma(t) \theta(t)=B(t)$, a.s., a.e.t $\in[0, T]$. Hence Assumption (A) is very close to the arbitrage-free assumption, a minimum condition for a "viable" market.

Let us now turn to the completeness of the market.

Definition 3. A contingent claim $\xi \in L_{\mathcal{F}_{T}}^{2}(\Omega ; \mathbf{R})$ is said to be replicable if there exists an initial wealth $x$ and an admissible wealth-portfolio pair $(x(\cdot), \pi(\cdot))$ satisfying $(3)$ with $x(T)=\xi$. The market is called complete if any contingent claim $\xi \in L_{\mathcal{F}_{T}}^{2}(\Omega ; \mathbf{R})$ is replicable.

Proposition 4. Under Assumption (A), the market is complete if and only if $\operatorname{rank}(\sigma(t))=n$, a.s., a.e. $t \in[0, T]$.

Proof. Consider the backward stochastic differential equation (BSDE) with a given $\xi \in L_{\mathcal{F}_{T}}^{2}(\Omega ; \mathbf{R})$ :

$$
d x(t)=\left[r(t) x(t)+\theta(t)^{\prime} z(t)\right] d t+z(t)^{\prime} d W(t), \quad x(T)=\xi,
$$

which admits a unique solution pair $(x(\cdot), z(\cdot)) \in L_{\mathcal{F}}^{2}(0, T, \mathbf{R}) \times L_{\mathcal{F}}^{2}\left(0, T, \mathbf{R}^{n}\right)$. If $\operatorname{rank}(\sigma(t))=n$, a.s., a.e.t $\in[0, T]$, then there exists $\pi(\cdot)$ such that $\sigma(t)^{\prime} \pi(t)=z(t)$, a.s., a.e.t $\in[0, T]$. By Lemma 28, we may assume that the process $\pi(\cdot)$ is $\mathcal{F}_{t}$-progressively measurable. Substituting $z(t)$ by $\sigma(t)^{\prime} \pi(t)$ in (9) we conclude that $(x(\cdot), \pi(\cdot))$ is an admissible wealth-portfolio pair with $x(T)=\xi$; hence $\xi$ is replicable. 
Conversely, assume that the market is complete. For any $z \in \mathbf{R}^{n}$, let $y(\cdot)$ solves the following SDE

$$
d y(t)=\left[r(t) y(t)+\theta(t)^{\prime} z\right] d t+z^{\prime} d W(t), \quad y(0)=0 .
$$

Since $y(T)$ is replicable, there exists $(x(\cdot), \pi(\cdot)) \in L_{\mathcal{F}}^{2}(0, T, \mathbf{R}) \times L_{\mathcal{F}}^{2}\left(0, T, \mathbf{R}^{m}\right)$ with $\sigma(\cdot)^{\prime} \pi(\cdot) \in L_{\mathcal{F}}^{2}\left(0, T, \mathbf{R}^{n}\right)$ so that

$$
d x(t)=\left[r(t) x(t)+\theta(t)^{\prime} \sigma(t)^{\prime} \pi(t)\right] d t+\pi(t)^{\prime} \sigma(t) d W(t), \quad x(T)=y(T) .
$$

Comparing the two preceding equations and by the uniqueness of the BSDE solution we conclude that $\sigma(t)^{\prime} \pi(t)=z$. This yields $\operatorname{rank}(\sigma(t))=n$ as $z \in \mathbf{R}^{n}$ is arbitrary.

Remark 5. There is a very similar result in [15, p. 24, Theorem 6.6]. However, notice that in [15] an admissible portfolio is defined to be square integrable in $t$ almost surely in $\omega$ and tame (i.e., the corresponding wealth process is bounded below), whereas in our mean-variance setting an admissible portfolio is required to be square integrable in $(t, \omega)$ (otherwise the variance of the terminal wealth may not even be well defined). Moreover, the definition of completeness is also different there in terms of the set of contingent claims to be replicated (see [15, p. 21, Definition 6.1]). In other words, we have a different class of admissible portfolios and a different notion of market completeness which are dictated by the nature of our problem.

It should be noted that the number of stocks, $m$, is generally different from the dimension of the underlying Brownian motion, $n$, and $\operatorname{rank}(\sigma(t))=n$ may not hold. Hence, the market is in general incomplete in our setup.

The following technical lemma is useful in the sequel.

Lemma 6. Given a set $A \subseteq L_{\mathcal{F}}^{\infty}\left(0, T, \mathbf{R}^{n}\right)$. If $k \theta_{1}+(1-k) \theta_{2} \in A$ whenever $\theta_{1} \in A, \theta_{2} \in A$ and $k \in L_{\mathcal{F}}^{1}(0, T ;[0,1])$, then the set $\left\{H_{\theta}(\cdot): \theta \in A\right\}$ is convex.

Proof. For any $\theta_{1}, \theta_{2} \in A$ and $\lambda \in[0,1]$, denote $H(\cdot):=\lambda H_{\theta_{1}}(\cdot)+(1-$ ג) $H_{\theta_{2}}(\cdot)$. Then $H(0)=1$, and

$$
\begin{aligned}
d H(t)= & \lambda\left[-r(t) H_{\theta_{1}}(t) d t-H_{\theta_{1}}(t) \theta_{1}(t)^{\prime} d W(t)\right] \\
& +(1-\lambda)\left[-r(t) H_{\theta_{2}}(t) d t-H_{\theta_{2}}(t) \theta_{2}(t)^{\prime} d W(t)\right] \\
= & -r(t) H(t) d t-H(t)\left[k(t) \theta_{1}(t)+(1-k(t)) \theta_{2}(t)\right]^{\prime} d W(t),
\end{aligned}
$$

where $k(t):=\frac{\lambda H_{\theta_{1}}(t)}{\lambda H_{\theta_{1}}(t)+(1-\lambda) H_{\theta_{2}}(t)}$. Define $\theta(t):=k(t) \theta_{1}(t)+[1-k(t)] \theta_{2}(t)$. Then $\theta \in A$. It then follows from the definition of $H_{\theta}$, see $(5)$, that $H(t) \equiv$ $H_{\theta}(t)$. This completes the proof.

To end this section we introduce two stochastic processes that are vital for the subsequent analysis.

Define the following processes 


$$
\theta^{*}(t):=\operatorname{argmin}_{\theta \in\left\{\theta \in \mathbf{R}^{n}: \sigma(t) \theta=B(t)\right\}}|\theta|^{2},
$$

and

$$
\hat{\theta}(t):=\operatorname{argmin}_{\theta \in\left\{\theta \in \mathbf{R}^{n}: \sigma(t) \theta \geq B(t)\right\}}|\theta|^{2} .
$$

Lemma 7. We have the following conclusions:

(i) $\theta^{*} \in L_{\mathcal{F}}^{\infty}\left(0, T, \mathbf{R}^{n}\right)$ and $\hat{\theta} \in L_{\mathcal{F}}^{\infty}\left(0, T, \mathbf{R}^{n}\right)$.

(ii) There exists an $\mathbf{R}^{m}$-valued, $\mathcal{F}_{t}$-progressively measurable process $u(\cdot)$ such that $\sigma(t)^{\prime} u(t)=\theta^{*}(t)$, a.s., a.e. $t \in[0, T]$.

(iii) There exists an $\mathbf{R}_{+}^{m}$-valued, $\mathcal{F}_{t}$-progressively measurable process $v(\cdot)$ such that $\sigma(t)^{\prime} v(t)=\hat{\theta}(t)$, a.s., a.e.t $\in[0, T]$.

(iv) For any $\theta \in \Theta, \theta^{*}(t)^{\prime} \theta(t)=\left|\theta^{*}(t)\right|^{2}$, a.s., a.e.t $\in[0, T]$.

(v) For any $\theta \in \hat{\Theta}, \hat{\theta}(t)^{\prime} \theta(t) \geq|\hat{\theta}(t)|^{2}=\hat{\theta}(t)^{\prime} \theta^{*}(t)$, a.s., a.e.t $\in[0, T]$.

The proof is relegated to the appendix.

Remark 8. When $\sigma(t)^{\prime} \sigma(t)$ is uniformly positive definite (in which case the market is complete) the process $\theta^{*}$ is the only $\theta$ that satisfies $\sigma(t) \theta(t)=B(t)$, and $\theta^{*}$ is the so-called pricing kernel. In the present case of incomplete market, as will be demonstrated in what follows, $\theta^{*}$ and $\hat{\theta}$ play the same important roles of pricing kernels associated with different constrains on portfolios.

\section{Markowitz's Portfolio Selection Models}

Fix an initial wealth $x_{0}$. A general continuous-time Markowitz's meanvariance portfolio selection problem (with constrained portfolios) is formulated as

$$
\begin{aligned}
& \text { minimize } \operatorname{Var} x(T) \equiv E x(T)^{2}-z^{2}, \\
& \text { subject to }\left\{\begin{array}{l}
\operatorname{Ex}(T)=z, \pi(\cdot) \in \Pi, \\
(x(\cdot), \pi(\cdot)) \text { satisfies equation }(3) \text { with } x(0)=x_{0}, \\
(x(\cdot), \pi(\cdot)) \in C,
\end{array}\right.
\end{aligned}
$$

where $C$ is a given convex set in $L_{\mathcal{F}}^{2}(0, T, \mathbf{R}) \times \Pi$, and $z \in \mathbf{R}$ is a parameter. The optimal portfolio for this problem (corresponding to a fixed $z$ ) is called an efficient portfolio, and the set of all points $\left(\operatorname{Var} x^{*}(T), z\right)$, where $\operatorname{Var} x^{*}(T)$ denotes the optimal value of (12) corresponding to $z$ and $z$ runs over certain range of $\mathbf{R}$, is called the efficient frontier.

Remark 9. For each $z$, an optimal solution to (12) in fact gives rise to a variance minimizing portfolio, and the set of all points $\left(\operatorname{Var} x^{*}(T), z\right)$ where $z$ runs over the whole real axis is called a variance minimizing frontier. The financial interpretation of a variance minimizing portfolio is clear: it tries to minimize the variance, representing the risk, while specifying a targeted expected return depicted by $z$. A main difference between the Markowitz model 
and the utility one, inter alia, is the presence of this constraint on the terminal payoff. On the other hand, in the original Markowitz's definition, an efficient portfolio is both variance minimizing and return maximizing (that is, it maximizes the expected terminal payoff subject to a same variance level). In other words, the efficient frontier is only a certain portion of the variance minimizing frontier. This is why in the above definition the efficient frontier only corresponds to $z$ being in certain range. There is a detailed study on this range in [2]. Here we only remark that in the case when all the market coefficients are deterministic, then the efficient range of $z$ is $z \geq x_{0} e^{\int_{0}^{T} r(t) d t}$.

In this paper, the following two cases of the constraint set $C$ will be studied respectively:

Case 1. $C=L_{\mathcal{F}}^{2}(0, T, \mathbf{R}) \times \Pi$, corresponding to the case where portfolios are not constrained.

Case 2. $C=\left\{(x(\cdot), \pi(\cdot)) \in L_{\mathcal{F}}^{2}(0, T, \mathbf{R}) \times \Pi: \pi(t) \geq 0\right.$, a.s., a.e.t $\left.\in[0, T]\right\}$, corresponding to the case where short-selling is prohibited.

Given a constraint set $C$ associated with one of the two cases above, define the following attainable terminal wealth set:

$$
\begin{aligned}
& A_{C}:=\left\{X \in L_{\mathcal{F}_{T}}^{2}(\Omega ; \mathbf{R}): \text { there exist } x \in \mathbf{R} \text { and } \pi(\cdot) \in \Pi\right. \text { such that } \\
& (x(\cdot), \pi(\cdot)) \text { satisfies }(3) \text { with } x(0)=x, x(T)=X, \text { and }(x(\cdot), \pi(\cdot)) \in C\} .
\end{aligned}
$$

To solve problem (12), the following static optimization problem plays a critical role:

$$
\begin{aligned}
\operatorname{minimize} & E X^{2}-z^{2}, \\
\text { subject to } & \left\{\begin{array}{l}
E X=z, \\
E\left[X H_{\theta^{*}}(T)\right]=x_{0}, \\
X \in A_{C},
\end{array}\right.
\end{aligned}
$$

where $\theta^{*}$ is defined by (10). This problem is to locate the optimal attainable terminal wealth $X^{*}$ in $A_{C}$. Once this is solved, an optimal portfolio for (12) can be obtained by replicating $X^{*}$ (which is possible by the very definition of $A_{C}$ along with the second constraint in (14)). Notice that, compared with the case of a complete market [2], the main difficulty in the present incomplete market situation is to characterize the attainable set $A_{C}$ for each of the two cases before solving (14).

The following result verifies that in order to solve the original problem (12) it suffices to solve (14).

Theorem 10. If $\left(x^{*}(\cdot), \pi^{*}(\cdot)\right)$ is optimal for (12), then $x^{*}(T)$ is optimal for (14). Conversely, if $X^{*} \in A_{C}$ is optimal for (14), then any wealth-portfolio pair $\left(x^{*}(\cdot), \pi^{*}(\cdot)\right)$ satisfying (3) with $\left(x^{*}(\cdot), \pi^{*}(\cdot)\right) \in C$ and $x^{*}(T)=X^{*}$ is optimal for (12). 
Proof. This is straightforward by the definition of $A_{C}$.

To solve (14), we first transform it to an equivalent problem as stipulated in the following theorem.

Theorem 11. If problem (14) admits a solution $X^{*}$, then there exists a pair of scalars $(\lambda, \mu)$ such that $X^{*}$ is also the optimal solution for the following problem:

$$
\begin{array}{ll}
\text { minimize } & E\left[X-\left(\lambda-\mu H_{\theta^{*}}(T)\right)\right]^{2}, \\
\text { subject to } & X \in A_{C} .
\end{array}
$$

Conversely, if there is a pair of scalars $(\lambda, \mu)$ such that the optimal solution $X^{*}$ of (15) satisfies

$$
\left\{\begin{array}{l}
E X^{*}=z, \\
E\left[X^{*} H_{\theta^{*}}(T)\right]=x_{0} .
\end{array}\right.
$$

then $X^{*}$ must be an optimal solution of (14).

Proof. It is easy to see that $A_{C}$ is a convex set due to the convexity of $C$ (for both cases). Hence the theorem can be proved in exactly the same fashion as [2, Theorem 4.1] (by applying a Lagrange multiplier approach [2, Proposition 4.1]).

The preceding theorem suggests that in order to solve (14) one can first solve problem (15) for general $(\lambda, \mu)$, which is a problem with $A_{C}$ being the only constraint set, and then determine the values of $(\lambda, \mu)$ via the equations (16).

To re-capture, solving the mean-variance problem (12) consists of the following steps:

Step 1 Solve (15) with parameters $(\lambda, \mu)$ and get solution $X^{*}=X^{*}(\lambda, \mu)$.

Step 2 Determine the values of $(\lambda, \mu)$ via (16).

Step 3 Any admissible portfolio (that satisfies the constraint specified by $C$ )

replicating $X^{*}(\lambda, \mu)$ is an efficient portfolio.

In the next two sections, we will study the two cases respectively. We will mainly devote ourselves to characterizing the attainable set $A_{C}$ and solving (15) for each case. For the general situation when the market parameters $r(\cdot), \mu_{i}(\cdot)$ and $\sigma_{i j}(\cdot)$ are stochastic processes, it is impossible to solve (15) explicitly in terms of $(\lambda, \mu)$. However, for the market when all the parameters are deterministic, we will obtain analytical solution to (15) and thereby get explicit solution to the original problem (12) for both cases.

\section{Case 1: Portfolios Unconstrained}

In this case the constraint set $C=L_{\mathcal{F}}^{2}(0, T, \mathbf{R}) \times \Pi$. Our first result characterizes the attainable terminal wealth set $A_{C}$ for this constraint set. 
Theorem 12. Given $X \in L_{\mathcal{F}_{T}}^{2}(\Omega ; \mathbf{R})$. The following assertions are equivalent:

(i) $X \in A_{C}$.

(ii) $E\left[X H_{\theta}(T)\right]$ is independent of $\theta \in \Theta$.

(iii) $E\left[X H_{\theta}(T)\right]$ is independent of $\theta \in \Theta_{1}$ where

$$
\Theta_{1}:=\left\{\theta \in \Theta:\left\|\theta-\theta^{*}\right\|_{L_{\mathcal{F}}^{\infty}\left(0, T, \mathbf{R}^{n}\right)} \leq 1\right\} .
$$

Proof. If $X \in A_{C}$, then there is $x \in \mathbf{R}$ and a portfolio $\pi(\cdot) \in \Pi$ such that

$$
\left\{\begin{array}{l}
d x(t)=\left[r(t) x(t)+B(t)^{\prime} \pi(t)\right] d t+\pi(t)^{\prime} \sigma(t) d W(t), \\
x(0)=x, \quad x(T)=X .
\end{array}\right.
$$

Now, for any $\theta \in \Theta$,

$$
\begin{aligned}
d x(t) & =\left[r(t) x(t)+B(t)^{\prime} \pi(t)\right] d t+\pi(t)^{\prime} \sigma(t) d W(t) \\
& =\left[r(t) x(t)+\theta(t)^{\prime} \sigma(t)^{\prime} \pi(t)\right] d t+\pi(t)^{\prime} \sigma(t) d W(t) .
\end{aligned}
$$

Applying Ito's formula, we obtain

$$
x \equiv x(0)=E\left[x(T) H_{\theta}(T)\right]=E\left[X H_{\theta}(T)\right],
$$

implying that $E\left[X H_{\theta}(T)\right]$ is independent of the choice of $\theta \in \Theta$. This proves that (i) implies (ii).

The implication from (ii) to (iii) is trivial. To close the loop of equivalence we prove that (iii) yields (i). Assume that $E\left[X H_{\theta}(T)\right]$ does not depend on $\theta \in \Theta_{1}$. By the BSDE theory, for any $\theta \in \Theta_{1}$, the following equation

$$
\left\{\begin{array}{l}
d X(t)=\left[r(t) X(t)+\theta(t)^{\prime} Z(t)\right] d t+Z(t)^{\prime} d W(t), \\
X(T)=X
\end{array}\right.
$$

admits a unique solution pair $\left(X_{\theta}(\cdot), Z_{\theta}(\cdot)\right)$, with $X_{\theta}(0)=E\left[X H_{\theta}(T)\right]$. So by the assumption $X_{\theta}(0), \theta \in \Theta_{1}$, are all the same, which is denoted by $x_{0}$.

Next, let $\left(X_{\theta^{*}}(\cdot), Z_{\theta^{*}}(\cdot)\right)$ solves (18) with $\theta=\theta^{*}$. We are to prove that there exists a portfolio $\pi_{0}(\cdot) \in \Pi$ such that

$$
Z_{\theta^{*}}(t)=\sigma(t)^{\prime} \pi_{0}(t), \text { a.s., a.e. } t \in[0, T] .
$$

Indeed, define

$$
\pi_{0}(t):=\operatorname{argmin}_{\pi \in \operatorname{argmin}_{\pi \in \mathbf{R}^{m}}\left|\sigma(t)^{\prime} \pi-Z_{\theta^{*}}(t)\right|^{2}}|\pi|^{2} .
$$

Notice that the set $\operatorname{argmin}_{\pi \in \mathbf{R}^{m}}\left|\sigma(t)^{\prime} \pi-Z_{\theta^{*}}(t)\right|^{2}$ is nonempty due to the Frank-Wolfe theorem (Lemma 26). Moreover, $\pi \in \operatorname{argmin}_{\pi \in \mathbf{R}^{m}} \mid \sigma(t)^{\prime} \pi-$ $\left.Z_{\theta^{*}}(t)\right|^{2}$ if and only if $\sigma(t) \sigma(t)^{\prime} \pi-\sigma(t) Z_{\theta^{*}}(t)=0$. Thus $\pi_{0}(t)$ is well-defined (again by the Frank-Wolfe theorem). Furthermore, we can apply Lemma 28 to conclude that $\pi_{0}(\cdot)$ is an $\mathcal{F}_{t}$-progressively measurable stochastic process.

Set $\bar{\rho}(t):=\sigma(t)^{\prime} \pi_{0}(t)-Z_{\theta^{*}}(t)$ and 


$$
\rho(t):= \begin{cases}0, & \text { if } \bar{\rho}(t)=0 \\ \bar{\rho}(t) /|\bar{\rho}(t)|, & \text { if } \bar{\rho}(t) \neq 0 .\end{cases}
$$

Then $\rho(\cdot) \in L_{\mathcal{F}}^{\infty}\left(0, T, \mathbf{R}^{n}\right)$. Moreover, $\sigma(t) \bar{\rho}(t)=\sigma(t) \sigma(t)^{\prime} \pi_{0}(t)-\sigma(t) Z_{\theta^{*}}(t)=$ 0 , owing to the fact that $\pi_{0}(t)$ minimizes $\left|\sigma(t)^{\prime} \pi-Z_{\theta^{*}}(t)\right|^{2}$. This implies that $\sigma(t) \rho(t)=0$ and hence

$$
\theta^{*}+\rho \in \Theta_{1} .
$$

On the other hand, $Z_{\theta^{*}}(t)^{\prime} \bar{\rho}(t)=\left[\pi_{0}(t)^{\prime} \sigma(t) \bar{\rho}(t)-\bar{\rho}(t)^{\prime} \bar{\rho}(t)\right]=-|\bar{\rho}(t)|^{2}$; thus $Z_{\theta^{*}}(t)^{\prime} \rho(t)=-|\bar{\rho}(t)|^{2}$.

Define $\hat{X}(\cdot)$ to be the solution of the following (forward) SDE:

$$
\left\{\begin{array}{l}
d \hat{X}(t)=\left[r(t) \hat{X}(t)+\left(\theta^{*}(t)+\rho(t)\right)^{\prime} Z_{\theta^{*}}(t)\right] d t+Z_{\theta^{*}}(t)^{\prime} d W(t), \\
\hat{X}(0)=x_{0}
\end{array}\right.
$$

Ito's formula implies

$$
E\left[\hat{X}(T) H_{\theta^{*}+\rho}(T)\right]=\hat{X}(0)=x_{0}=E\left[X_{\theta^{*}}(T) H_{\theta^{*}+\rho}(T)\right],
$$

where the last equality is due to (20) and the assumption. However,

$d\left[\hat{X}(t)-X_{\theta^{*}}(t)\right]=r(t)\left[\hat{X}(t)-X_{\theta^{*}}(t)\right] d t+Z_{\theta^{*}}(t)^{\prime} \rho(t) d t, \hat{X}(0)-X_{\theta^{*}}(0)=0 ;$

hence $\hat{X}(T)-X_{\theta^{*}}(T)=\int_{0}^{T} e^{\int_{t}^{T} r(s) d s} Z_{\theta^{*}}(t)^{\prime} \rho(t) d t=-\int_{0}^{T} e^{\int_{t}^{T} r(s) d s}|\bar{\rho}(t)|^{2} d t$. Comparing this with $(21)$ we conclude that $\bar{\rho}(t)=0$, a.s., a.e.t $\in[0, T]$, which leads to (19). Since $\sigma(\cdot)^{\prime} \pi_{0}(\cdot)=Z_{\theta^{*}}(\cdot) \in L_{\mathcal{F}}^{2}\left(0, T, \mathbf{R}^{n}\right)$, it follows that $\pi_{0}(\cdot) \in \Pi$. Now, the BSDE (18) that $\left(X_{\theta^{*}}(\cdot), Z_{\theta^{*}}(\cdot)\right)$ satisfies can be rewritten as

$$
\left\{\begin{array}{l}
d X_{\theta^{*}}(t)=\left[r(t) X_{\theta^{*}}(t)+B(t)^{\prime} \pi_{0}(t)\right] d t+\pi_{0}(t)^{\prime} \sigma(t) d W(t), \\
X_{\theta^{*}}(T)=X
\end{array}\right.
$$

which means that $X$ is attained by the portfolio $\pi_{0}(\cdot)$.

Remark 13. Similar results have been obtained before in, e.g., $[8,6,16]$, albeit in different contexts. Again, in these works, an admissible portfolio is defined to be square integrable in $t$ almost surely in $\omega$ and/or tame, which is different from ours. There are also other technical subtleties comparing our result to the existing ones. For example, in [8] a contingent claim is assumed to be bounded above by the terminal value of a portfolio (see [8, p. 35]). This condition seems to be critical in deriving the result there. Finally, we believe our proof, based on the BSDE theory, is quite clean and simple compared with those in $[8,6,16]$. The same can be said of Theorem 20 for the no-shorting case.

Corollary 14. $A_{C}$ is a (nonempty) linear subspace of $L_{\mathcal{F}_{T}}^{2}(\Omega ; \mathbf{R})$.

By Theorem 12, we can rewrite problem (15) as follows: 


$$
\begin{array}{ll}
\operatorname{minimize} & E\left[X-\left(\lambda-\mu H_{\theta^{*}}(T)\right)\right]^{2}, \\
\text { subject to } & \left\{\begin{array}{l}
X \in L_{\mathcal{F}_{T}}^{2}(\Omega ; \mathbf{R}), \\
E\left[X\left(H_{\theta}(T)-H_{\theta^{*}}(T)\right)\right]=0 \quad \forall \theta \in \Theta .
\end{array}\right.
\end{array}
$$

First notice that (22) is a convex optimization problem with a coercive, strictly convex cost function and a nonempty, closed convex constraint set; hence it must admit a unique optimal solution. However, it is generally hard to construct the optimal solution since (22) actually involves infinitely many constraints. Denote $L:=\operatorname{span}\left\{H_{\theta}(T)-H_{\theta^{*}}(T): \theta \in \Theta\right\}$, where $\operatorname{span}(A)$ means the minimal linear space that contains $A$, and consider $\bar{L}$, the closure of $L$ in the $L_{\mathcal{F}_{T}}^{2}(\Omega ; \mathbf{R})$-norm. Since each $H_{\theta}(T) \in L_{\mathcal{F}_{T}}^{2}(\Omega ; \mathbf{R})$, it follows that $\bar{L} \subset L_{\mathcal{F}_{T}}^{2}(\Omega ; \mathbf{R})$. The following theorem provides a way to finding a solution to $(22)$.

Theorem 15. For any given $(\lambda, \mu)$, consider the following problem

$$
\begin{aligned}
& \text { minimize } E\left(\lambda-\mu H_{\theta^{*}}(T)-Y\right)^{2}, \\
& \text { subject to } Y \in \bar{L} \text {. }
\end{aligned}
$$

We have the following conclusions:

(i) Problem (23) admits a unique optimal solution. Moreover, $Y^{*} \in \bar{L}$ is the optimal solution to (23) if and only if $\lambda-\mu H_{\theta^{*}}(T)-Y^{*} \in A_{C}$.

(ii) The unique optimal solution to (22) can be expressed as $X^{*}=\lambda$ $\mu H_{\theta^{*}}(T)-Y^{*}$ where $Y^{*}$ is the unique optimal solution to (23).

Proof. (i) First of all, by the projection theorem in Hilbert spaces (refer to, e.g., [22, p.51, Theorem 2]), (23) has a unique optimal solution $Y^{*}$. Moreover, $Y^{*}$ is optimal for (23) if and only if $E\left[\left(\lambda-\mu H_{\theta^{*}}(T)-Y^{*}\right) Y\right]=0 \forall Y \in \bar{L}$. The latter is equivalent to that $X^{*}:=\lambda-\mu H_{\theta^{*}}(T)-Y^{*}$ is feasible for (22) which, in view of Theorem 12 , is further equivalent to that $\lambda-\mu H_{\theta^{*}}(T)-Y^{*} \in A_{C}$.

(ii) We have proved in (i) that $X^{*}=\lambda-\mu H_{\theta^{*}}(T)-Y^{*}$ is feasible for (22) if $Y^{*}$ is optimal for (23). Now, for any feasible solution $X$ of (22):

$$
\begin{aligned}
E[X-(\lambda & \left.\left.-\mu H_{\theta^{*}}(T)\right)\right]^{2} \\
= & E\left[X-\left(\lambda-\mu H_{\theta^{*}}(T)\right)+Y^{*}\right]^{2} \\
& +2 E\left[Y^{*}\left(\lambda-\mu H_{\theta^{*}}(T)\right)\right]-2 E\left[X Y^{*}\right]-E\left[Y^{*}\right]^{2} \\
= & E\left[X-\left(\lambda-\mu H_{\theta^{*}}(T)-Y^{*}\right)\right]^{2} \\
& +2 E\left[Y^{*}\left(\lambda-\mu H_{\theta^{*}}(T)\right)\right]-2 E\left[X^{*} Y^{*}\right]-E\left[Y^{*}\right]^{2} \\
\geq & E\left[X^{*}-\left(\lambda-\mu H_{\theta^{*}}(T)-Y^{*}\right)\right]^{2} \\
& +2 E\left[Y^{*}\left(\lambda-\mu H_{\theta^{*}}(T)\right)\right]-2 E\left[X^{*} Y^{*}\right]-E\left[Y^{*}\right]^{2} \\
= & E\left[X^{*}-\left(\lambda-\mu H_{\theta^{*}}(T)\right)\right]^{2},
\end{aligned}
$$

where we have used the fact that $E\left[X Y^{*}\right]=E\left[X^{*} Y^{*}\right]=0$ due to the constraint of problem $(22)$. Hence $X^{*}$ is the unique optimal solution to (22). 
Remark 16. The above theorem suggests that one can obtain the optimal solution to (22), hence that to (15), via the (unique) optimal solution to the projection problem (23). In fact, (23) is a dual problem of (22), in the sense that the cost function of the former is the conjugate of that of the latter, while the feasible regions of the two problems are orthogonal to each other. In many cases the primal-dual relation between (22) and (23) helps us in finding solutions to the both, as solving one problem may be easier than directly solving the other.

When the market parameters, $r(\cdot), \mu(\cdot)$ and $\sigma(\cdot)$, are all deterministic processes, both (22) and (23) can be solved explicitly which in turn leads to the closed-form solution to the underlying mean-variance portfolio selection problem.

Lemma 17. If $r(\cdot), \mu(\cdot)$ and $\sigma(\cdot)$ are deterministic, then $\lambda-\mu H_{\theta^{*}}(T) \in A_{C}$ for any $(\lambda, \mu)$.

Proof. Fix $\theta \in \Theta$. We have

$$
\begin{aligned}
H_{\theta^{*}} & (T) H_{\theta}(T) \\
& =\exp \left\{-\int_{0}^{T}\left[2 r(t)+\frac{1}{2}\left(|\theta(t)|^{2}+\left|\theta^{*}(t)\right|^{2}\right)\right] d t-\int_{0}^{T}\left[\theta(t)+\theta^{*}(t)\right]^{\prime} d W(t)\right\} \\
& =\exp \left\{-\int_{0}^{T}\left[2 r(t)-\left|\theta^{*}(t)\right|^{2}\right] d t\right\} \\
& \times \exp \left\{-\int_{0}^{T} \frac{1}{2}\left|\theta(t)+\theta^{*}(t)\right|^{2} d t-\int_{0}^{T}\left[\theta(t)+\theta^{*}(t)\right]^{\prime} d W(t)\right\},
\end{aligned}
$$

where we have used $\theta^{*}(t)^{\prime} \theta(t)=\left|\theta^{*}(t)\right|^{2}$; see Lemma 7-(iv). Thus,

$$
E\left[H_{\theta^{*}}(T) H_{\theta}(T)\right]=\exp \left\{-\int_{0}^{T}\left[2 r(t)-\left|\theta^{*}(t)\right|^{2}\right] d t\right\}
$$

which is independent of $\theta \in \Theta$. We have then $H_{\theta^{*}}(T) \in A_{C}$ thanks to Theorem 12. The conclusion follows as $\lambda \in A_{C}$.

Theorem 18. If $r(\cdot), \mu(\cdot)$ and $\sigma(\cdot)$ are deterministic, and $\int_{0}^{T}|B(t)| d t>0$, then $\pi(t):=\left[\lambda e^{-\int_{t}^{T} r(s) d s}-x(t)\right] u(t)$ is an efficient portfolio, in a feedback form, for the mean-variance problem (12) corresponding to $z \geq x_{0} e^{\int_{0}^{T} r(t) d t}$, where

$$
\lambda=\frac{z e^{\int_{0}^{T}\left|\theta^{*}(t)\right|^{2} d t}-x_{0} e^{\int_{0}^{T} r(t) d t}}{e^{\int_{0}^{T}\left|\theta^{*}(t)\right|^{2} d t}-1}, \quad \mu=\frac{z e^{\int_{0}^{T} r(t) d t}-x_{0} e^{\int_{0}^{T} 2 r(t) d t}}{e^{\int_{0}^{T}\left|\theta^{*}(t)\right|^{2} d t}-1}
$$

and $u(\cdot)$ is a measurable function satisfying $\sigma(t)^{\prime} u(t)=\theta^{*}(t)$. Moreover, the efficient frontier is 


$$
\operatorname{Var}(x(T))=\frac{1}{e^{\int_{0}^{T}\left|\theta^{*}(t)\right|^{2} d t}-1}\left[z-x_{0} e^{\int_{0}^{T} r(t) d t}\right]^{2}, \quad z \geq x_{0} e^{\int_{0}^{T} r(t) d t} .
$$

Proof. Fix $z \geq x_{0} e^{\int_{0}^{T} r(t) d t}$. By virtue of Lemma 17 and Theorem 15, $Y^{*}=$ 0 is the unique optimal solution to (23), or $X^{*}=\lambda-\mu H_{\theta^{*}}(T)$ is the unique optimal solution to $(22)$. To determine $(\lambda, \mu)$ so as to obtain the solution to (14), we apply Theorem 11 to derive the following system of equations

$$
\left\{\begin{array}{l}
\lambda-\mu E H_{\theta^{*}}(T)=z \\
\lambda E H_{\theta^{*}}(T)-\mu E\left[H_{\theta^{*}}(T)^{2}\right]=x_{0} .
\end{array}\right.
$$

It follows from $\int_{0}^{T}|B(t)| d t>0$ that $e^{\int_{0}^{T}\left|\theta^{*}(t)\right|^{2} d t}-1 \neq 0$. Solving the preceding equations we get the expressions (24), noting that $E H_{\theta^{*}}(T)=$ $e^{-\int_{0}^{T} r(t) d t}, E\left[H_{\theta^{*}}(T)^{2}\right]=e^{-\int_{0}^{T}\left[2 r(t)-\left|\theta^{*}(t)\right|^{2}\right] d t}$.

An admissible portfolio is efficient corresponding to $z$ if it replicates the terminal wealth $X^{*}=\lambda-\mu H_{\theta^{*}}(T)$. Appealing to (8), we can get the corresponding wealth process to be

$$
\begin{aligned}
x(t)=H_{\theta^{*}}(t)^{-1} E\left(\left[\lambda-\mu H_{\theta^{*}}(T)\right] H_{\theta^{*}}(T) \mid \mathcal{F}_{t}\right) & \\
& =\lambda e^{-\int_{t}^{T} r(s) d s}-\mu e^{-\int_{t}^{T}\left(2 r(s)-\left|\theta^{*}(s)\right|^{2}\right) d s} H_{\theta^{*}}(t) .
\end{aligned}
$$

A direct computation on the above, using (5), yields

$$
\begin{aligned}
d x(t)= & {\left[r x(t)+\mu\left|\theta^{*}(t)\right|^{2} e^{-\int_{t}^{T}\left(2 r(s)-\left|\theta^{*}(s)\right|^{2}\right) d s} H_{\theta^{*}}(t)\right] d t } \\
& +\mu e^{-\int_{t}^{T}\left(2 r(s)-\left|\theta^{*}(s)\right|^{2}\right) d s} H_{\theta^{*}}(t) \theta^{*}(t)^{\prime} d W(t) \\
= & {\left[r x(t)+\mu e^{-\int_{t}^{T}\left(2 r(s)-\left|\theta^{*}(s)\right|^{2}\right) d s} H_{\theta^{*}}(t) \theta^{*}(t)^{\prime} \theta^{*}(t)\right] d t } \\
& +\mu e^{-\int_{t}^{T}\left(2 r(s)-\left|\theta^{*}(s)\right|^{2}\right) d s} H_{\theta^{*}}(t) \theta^{*}(t)^{\prime} d W(t) .
\end{aligned}
$$

Comparing the above with the wealth equation (3), we conclude that a portfolio $\pi(\cdot)$ realizes the wealth process $x(\cdot)$ if and only if

$$
\sigma(t)^{\prime} \pi(t)=\mu e^{-\int_{t}^{T}\left(2 r(s)-\left|\theta^{*}(s)\right|^{2}\right) d s} H_{\theta^{*}}(t) \theta^{*}(t) .
$$

By Lemma 7 -(ii), there exists an $\mathcal{F}_{t}$-progressively measurable process $u(\cdot)$ satisfying $\sigma(t)^{\prime} u(t)=\theta^{*}(t)$. Hence, the following portfolio

$$
\pi(t):=\mu e^{-\int_{t}^{T}\left(2 r(s)-\left|\theta^{*}(s)\right|^{2}\right) d s} H_{\theta^{*}}(t) u(t) \equiv\left[\lambda e^{-\int_{t}^{T} r(s) d s}-x(t)\right] u(t)
$$

indeed satisfies (26), and hence is efficient.

Finally, the variance of the optimal terminal wealth is

$$
\begin{aligned}
\operatorname{Var}(x(T))=\operatorname{Var}\left(X^{*}\right)=\mu^{2} \operatorname{Var}\left(H_{\theta^{*}}(T)\right)=\frac{\left[z E H_{\theta^{*}}(T)-x_{0}\right]^{2}}{\operatorname{Var}\left(H_{\theta^{*}}(T)\right)} & \\
= & \frac{1}{e^{\int_{0}^{T}\left|\theta^{*}(t)\right|^{2} d t}-1}\left[z-x_{0} e^{\int_{0}^{T} r(t) d t}\right]^{2} .
\end{aligned}
$$


Remark 19. While the wealth process that replicates $X^{*}$ is unique, there may be more than one replicating portfolios, i.e., there may be many portfolios $\pi(\cdot)$ satisfying (26). Hence, efficient portfolios corresponding to a same $z$ are not unique.

\section{Case 2: Shorting Prohibited}

Again, we need to first characterize the attainable set $A_{C}$ in this case.

Theorem 20. For any $X \in L_{\mathcal{F}_{T}}^{2}(\Omega ; \mathbf{R}), X \in A_{C}$ if and only if there exists $\bar{\theta} \in \hat{\Theta}$ such that $\sup _{\theta \in \hat{\Theta}} E\left[X H_{\theta}(T)\right]=E\left[X H_{\bar{\theta}}(T)\right]$. Furthermore, $\sup _{\theta \in \hat{\Theta}} E\left[X H_{\theta}(T)\right]=E\left[X H_{\theta^{*}}(T)\right]$ if $X \in A_{C}$.

Proof. If $X \in A_{C}$, then there is $(x(\cdot), \pi(\cdot)) \in C$ satisfying (3) with $x_{0}=$ $E\left[X H_{\theta^{*}}(T)\right]$. Take any $\theta \in \hat{\Theta}$ and consider $H_{\theta}(\cdot)$ that satisfies (5). Applying Ito's formula we get easily

$d\left[x(t) H_{\theta}(t)\right]=[B(t)-\sigma(t) \theta(t)]^{\prime} \pi(t) H_{\theta}(t) d t+\left[\pi(t)^{\prime} \sigma(t)-x(t) \theta(t)^{\prime}\right] H_{\theta}(t) d W(t) ;$

thus $E\left[X H_{\theta}(T)\right]=x_{0}+E \int_{0}^{T}[B(t)-\sigma(t) \theta(t)]^{\prime} \pi(t) H_{\theta}(t) d t \leq x_{0}=E\left[X H_{\theta^{*}}(T)\right]$ (here that the expectation of the stochastic integral vanishes can be proved in the same way as in proving (8); see [7, p. 22, Proposition 2.2]). This yields $\sup _{\theta \in \hat{\Theta}} E\left[X H_{\theta}(T)\right]=E\left[X H_{\theta^{*}}(T)\right]$.

Conversely, suppose there is $\bar{\theta} \in \hat{\Theta}$ such that $x_{0}:=E\left[X H_{\bar{\theta}}(T)\right] \geq$ $E\left[X H_{\theta}(T)\right] \forall \theta \in \hat{\Theta}$. Let $\left(X^{*}(\cdot), Z^{*}(\cdot)\right)$ be the unique solution to the following BSDE

$$
\left\{\begin{array}{l}
d X^{*}(t)=\left[r(t) X^{*}(t)+\bar{\theta}(t)^{\prime} Z^{*}(t)\right] d t+Z^{*}(t)^{\prime} d W(t) \\
X^{*}(T)=X
\end{array}\right.
$$

We are to show that there exists an admissible portfolio $\pi_{0}(\cdot)$ satisfying the no-shorting constraint such that

$$
Z^{*}(t)=\sigma(t)^{\prime} \pi_{0}(t), \text { a.s., a.e. } t \in[0, T] .
$$

Indeed, define

$$
\pi_{0}(t):=\operatorname{argmin}_{\pi \in \operatorname{argmin}_{\pi \in \mathbf{R}_{+}^{m}}\left|\sigma(t)^{\prime} \pi-Z^{*}(t)\right|^{2}}|\pi|^{2} .
$$

Note that $\operatorname{argmin}_{\pi \in \mathbf{R}_{+}^{m}}\left|\sigma(t)^{\prime} \pi-Z^{*}(t)\right|^{2} \neq \emptyset$ due to the Frank-Wolfe theorem (Lemma 26). On the other hand, $\pi \in \operatorname{argmin}_{\pi \in \mathbf{R}_{+}^{m}}\left|\sigma(t)^{\prime} \pi-Z^{*}(t)\right|^{2}$ can be rewritten as $\left|\sigma(t)^{\prime} \pi-Z^{*}(t)\right|^{2}-g(t) \leq 0$, where $g(t):=\min _{\pi \in \mathbf{R}_{+}^{m}} \mid \sigma(t)^{\prime} \pi-$ $\left.Z^{*}(t)\right|^{2}$ which is clearly $\mathcal{F}_{t}$-progressively measurable. Hence we can apply Lemma 28 to conclude that $\pi_{0}(\cdot)$ is an $\mathcal{F}_{t}$-progressively measurable stochastic process. Note $Z^{*}(t) \notin\left\{\sigma(t)^{\prime} \pi: \pi \in \mathbf{R}_{+}^{m}\right\}$ whenever $\sigma(t)^{\prime} \pi_{0}(t)-Z^{*}(t) \neq 0$. Thus by Lemmas 27 and 28 , there is $\bar{\rho}(\cdot)$ which is $\mathcal{F}_{t}$-progressively measurable 
satisfying $\bar{\rho}(t) \neq 0, Z^{*}(t)^{\prime} \bar{\rho}(t)<0, \sigma(t) \bar{\rho}(t) \geq 0$, a.s., a.e.t on the set where $\sigma(t)^{\prime} \pi_{0}(t)-Z^{*}(t) \neq 0$. Set

$$
\rho(t):= \begin{cases}0, & \text { if } \sigma(t)^{\prime} \pi_{0}(t)-Z^{*}(t)=0 \\ \bar{\rho}(t) /|\bar{\rho}(t)|, & \text { if } \sigma(t)^{\prime} \pi_{0}(t)-Z^{*}(t) \neq 0 .\end{cases}
$$

Then $\rho(\cdot) \in L_{\mathcal{F}}^{\infty}\left(0, T, \mathbf{R}^{n}\right), \sigma(t) \rho(t) \geq 0$, and

$$
Z^{*}(t)^{\prime} \rho(t)<0 \quad \text { whenever } \sigma(t)^{\prime} \pi_{0}(t)-Z^{*}(t) \neq 0 .
$$

Since $\sigma(t)[\bar{\theta}(t)+\rho(t)] \geq \sigma(t) \bar{\theta}(t) \geq B(t)$, we conclude $\bar{\theta}+\rho \in \hat{\Theta}$.

Define $\bar{X}(\cdot)$ to be the solution of the following SDE:

$$
\left\{\begin{array}{l}
d \bar{X}(t)=\left[r(t) \bar{X}(t)+(\bar{\theta}(t)+\rho(t))^{\prime} Z^{*}(t)\right] d t+Z^{*}(t)^{\prime} d W(t), \\
\bar{X}(0)=x_{0}
\end{array}\right.
$$

Then

$$
E\left[\bar{X}(T) H_{\bar{\theta}+\rho}(T)\right]=\bar{X}(0)=x_{0}=E\left[X^{*}(T) H_{\bar{\theta}}(T)\right] \geq E\left[X^{*}(T) H_{\bar{\theta}+\rho}(T)\right] .
$$

On the other hand,

$$
d\left[\bar{X}(t)-X^{*}(t)\right]=r(t)\left[\bar{X}(t)-X^{*}(t)\right] d t+Z^{*}(t)^{\prime} \rho(t) d t, \bar{X}(0)-X^{*}(0)=0 ;
$$

hence $\bar{X}(T)-X^{*}(T)=\int_{0}^{T} e^{\int_{t}^{T} r(s) d s} Z^{*}(t)^{\prime} \rho(t) d t$. It then follows from (31) and (30) that $\sigma(t)^{\prime} \pi_{0}(t)-Z^{*}(t)=0$, a.s., a.e. $t \in[0, T]$. This proves (28).

Next, let $\hat{X}(\cdot)$ be the solution to the following SDE:

$$
\left\{\begin{array}{l}
d \hat{X}(t)=\left[r(t) \hat{X}(t)+\theta^{*}(t)^{\prime} Z^{*}(t)\right] d t+Z^{*}(t)^{\prime} d W(t), \\
\hat{X}(0)=x_{0}
\end{array}\right.
$$

Then $E\left[\hat{X}(T) H_{\theta^{*}}(T)\right]=x_{0} \geq E\left[X^{*}(T) H_{\theta^{*}}(T)\right]$. On the other hand,

$$
\begin{aligned}
d\left[\hat{X}(t)-X^{*}(t)\right] & =r(t)\left[\hat{X}(t)-X^{*}(t)\right] d t+\left[\theta^{*}(t)-\bar{\theta}(t)\right]^{\prime} Z^{*}(t) d t \\
& =r(t)\left[\hat{X}(t)-X^{*}(t)\right] d t+[B(t)-\sigma(t) \bar{\theta}(t)]^{\prime} \pi_{0}(t) d t,
\end{aligned}
$$

where we have used the fact that $Z^{*}(t)=\sigma(t)^{\prime} \pi_{0}(t)$. Hence, $\hat{X}(T)-X^{*}(T)=$ $\int_{0}^{T} e^{\int_{t}^{T} r(s) d s}[B(t)-\sigma(t) \bar{\theta}(t)]^{\prime} \pi_{0}(t) d t \leq 0$. By $E\left[\left(\hat{X}(T)-X^{*}(T)\right) H_{\theta^{*}}(T)\right] \geq 0$ we have

$$
B(t)^{\prime} \pi_{0}(t)=\bar{\theta}(t)^{\prime} \sigma(t)^{\prime} \pi_{0}(t) \equiv \bar{\theta}(t)^{\prime} Z^{*}(t), \text { a.s., a.e. } t \in[0, T],
$$

and

$$
E\left[X H_{\bar{\theta}}(T)\right] \equiv x_{0} \equiv E\left[\hat{X}(T) H_{\theta^{*}}(T)\right]=E\left[X H_{\theta^{*}}(T)\right] .
$$

It follows from $(27)$ and $(33)$ that $\left(X^{*}(\cdot), \pi_{0}(\cdot)\right)$ satisfies

$$
\left\{\begin{array}{l}
d X^{*}(t)=\left[r(t) X^{*}(t)+B(t)^{\prime} \pi_{0}(t)\right] d t+\pi_{0}(t)^{\prime} \sigma(t) d W(t), \\
X^{*}(T)=X,
\end{array}\right.
$$

meaning that $X \in A_{C}$. Finally, the second assertion of the theorem follows from (34). 
Corollary 21. $A_{C}$ is a (nonempty) convex subset of $L_{\mathcal{F}_{T}}^{2}(\Omega ; \mathbf{R})$.

Remark 22. The preceding theorem along with Theorem 12 imply that $X \in$ $A_{C}$ if and only if the maximum of $E\left[X H_{\theta}(T)\right]$ over $\theta \in \hat{\Theta}$ is achieved at any point on $\Theta$, the "boundary" of $\hat{\Theta}$.

By virtue of Theorem 20, problem (15) for Case 2 can be written as

$$
\begin{array}{ll}
\operatorname{minimize} & E\left[X-\left(\lambda-\mu H_{\theta^{*}}(T)\right)\right]^{2}, \\
\text { subject to } & \left\{\begin{array}{l}
X \in L_{\mathcal{F}_{T}}^{2}(\Omega ; \mathbf{R}), \\
\max _{\theta \in \hat{\Theta}} E\left[X H_{\theta}(T)\right]=E\left[X H_{\theta^{*}}(T)\right] .
\end{array}\right.
\end{array}
$$

Denote $M:=\left\{k\left(H_{\theta}(T)-H_{\theta^{*}}(T)\right) \in L_{\mathcal{F}_{T}}^{2}(\Omega ; \mathbf{R}): k \geq 0, \theta \in \hat{\Theta}\right\}$, which can be easily verified, via Lemma 6 , to be a convex cone. Consider $\bar{M}$, the closure of $M$ in the $L_{\mathcal{F}_{T}}^{2}(\Omega ; \mathbf{R})$-norm, which is a closed convex cone.

The following theorem is the no-shorting counterpart of Theorem 15.

Theorem 23. For any given $(\lambda, \mu)$, consider the following problem

$$
\begin{aligned}
& \text { minimize } E\left(\lambda-\mu H_{\theta^{*}}(T)-Y\right)^{2}, \\
& \text { subject to } Y \in \bar{M} \text {. }
\end{aligned}
$$

We have the following conclusions:

(i) Problem (36) admits a unique optimal solution. Moreover, $Y^{*} \in \bar{M}$ is the optimal solution to (36) if and only if

$$
E\left[\left(\lambda-\mu H_{\theta^{*}}(T)-Y^{*}\right) Y^{*}\right]=0, \quad \lambda-\mu H_{\theta^{*}}(T)-Y^{*} \in A_{C} .
$$

(ii) The unique optimal solution to (35) can be expressed as $X^{*}=\lambda-$ $\mu H_{\theta^{*}}(T)-Y^{*}$ where $Y^{*}$ is the unique optimal solution to (36).

Proof. (i) First of all, (36) is an optimization problem with a coercive, strictly convex cost function and a nonempty, closed convex constraint set, which therefore must admit a unique optimal solution. Moreover, $Y^{*} \in \bar{M}$ is optimal to (36) if and only if for any $Y \in \bar{M}, 0 \in \operatorname{argmin}_{0 \leq \alpha \leq 1} E[f(\alpha)]$ where $f(\alpha):=\left[\lambda-\mu H_{\theta^{*}}(T)-Y^{*}+\alpha\left(Y^{*}-Y\right)\right]^{2}$. Now, for any sufficiently small $h>0$, we have

$$
\begin{aligned}
\left|\frac{1}{h}[f(h)-f(0)]\right| & \leq \frac{1}{h}\left|\left[2\left(\lambda-\mu H_{\theta^{*}}(T)-Y^{*}\right)+h\left(Y^{*}-Y\right)\right] h\left(Y^{*}-Y\right)\right| \\
& \leq\left|2\left(\lambda-\mu H_{\theta^{*}}(T)-Y^{*}\right)+h\left(Y^{*}-Y\right)\right| \cdot\left|Y^{*}-Y\right| .
\end{aligned}
$$

Thus by the dominated convergence theorem we have

$$
\begin{aligned}
\lim _{h \rightarrow 0+} \frac{E f(h)-E f(0)}{h} & =\left.E \frac{\partial}{\partial \alpha}\left[\lambda-\mu H_{\theta^{*}}(T)-Y^{*}+\alpha\left(Y^{*}-Y\right)\right]^{2}\right|_{\alpha=0} \\
& =2 E\left[\left(\lambda-\mu H_{\theta^{*}}(T)-Y^{*}\right)\left(Y^{*}-Y\right)\right] .
\end{aligned}
$$


Consequently, $Y^{*} \in \bar{M}$ is optimal if and only if

$$
E\left[\left(\lambda-\mu H_{\theta^{*}}(T)-Y^{*}\right)\left(Y^{*}-Y\right)\right] \geq 0 \quad \forall Y \in \bar{M} .
$$

To prove that (37) and (38) are equivalent, first note that (37) easily yields (38) thanks to Theorem 20. Now, suppose (38) holds. Taking $Y=0 \in \bar{M}$ we get from (38) that $E\left[\left(\lambda-\mu H_{\theta^{*}}(T)-Y^{*}\right) Y^{*}\right] \geq 0$, and taking $Y=2 Y^{*} \in \bar{M}$ (recall that $\bar{M}$ is a cone) we get $E\left[\left(\lambda-\mu H_{\theta^{*}}(T)-Y^{*}\right) Y^{*}\right] \leq 0$. Consequently $E\left[\left(\lambda-\mu H_{\theta^{*}}(T)-Y^{*}\right) Y^{*}\right]=0$ and, together with (38), results in (37).

(ii) We have proved in (i) that $X^{*}=\lambda-\mu H_{\theta^{*}}(T)-Y^{*}$ is feasible for (35) if $Y^{*}$ is optimal for (36). On the other hand, for any feasible solution $X$ of $(35)$ :

$$
\begin{aligned}
E[X-(\lambda & \left.\left.-\mu H_{\theta^{*}}(T)\right)\right]^{2} \\
= & \left.E\left[X-\left(\lambda-\mu H_{\theta^{*}}(T)\right)+Y^{*}\right)\right]^{2} \\
& +2 E\left[Y^{*}\left(\lambda-\mu H_{\theta^{*}}(T)\right)\right]-2 E\left[X Y^{*}\right]-E\left[Y^{*}\right]^{2} \\
\geq & E\left[X-\left(\lambda-\mu H_{\theta^{*}}(T)-Y^{*}\right)\right]^{2} \\
& +2 E\left[Y^{*}\left(\lambda-\mu H_{\theta^{*}}(T)\right)\right]-2 E\left[X^{*} Y^{*}\right]-E\left[Y^{*}\right]^{2} \\
\geq & E\left[X^{*}-\left(\lambda-\mu H_{\theta^{*}}(T)-Y^{*}\right)\right]^{2} \\
& +2 E\left[Y^{*}\left(\lambda-\mu H_{\theta^{*}}(T)\right)\right]-2 E\left[X^{*} Y^{*}\right]-E\left[Y^{*}\right]^{2} \\
= & E\left[X^{*}-\left(\lambda-\mu H_{\theta^{*}}(T)\right)\right]^{2},
\end{aligned}
$$

where we have used the facts that $E\left[X Y^{*}\right] \leq 0$ and $E\left[X^{*} Y^{*}\right]=0$. This means that $X^{*}$ is an optimal solution for (35).

As with Case 1, we now discuss the case when all the market coefficients are deterministic and show how to apply Theorem 23 to solve the mean-variance problem. First recall the definition of the pricing kernel $\hat{\theta}$; see (11).

Lemma 24. If $r(\cdot), \mu(\cdot)$ and $\sigma(\cdot)$ are deterministic, then for any given $(\lambda, \mu)$ with $\mu \geq 0, \lambda-\mu H_{\hat{\theta}}(T) \in A_{C}$ and (36) has the optimal solution $Y^{*}:=$ $\mu\left(H_{\hat{\theta}}(T)-H_{\theta^{*}}(T)\right)$.

Proof. According to Theorems 23 and 20, to prove the desired results it suffices to show that

$$
\begin{aligned}
& E\left[\left(\lambda-\mu H_{\hat{\theta}}(T)\right)\left(H_{\hat{\theta}}(T)-H_{\theta^{*}}(T)\right)\right]=0, \\
& E\left[\left(\lambda-\mu H_{\hat{\theta}}(T)\right)\left(H_{\theta}(T)-H_{\theta^{*}}(T)\right)\right] \leq 0 \quad \forall \theta \in \hat{\Theta} .
\end{aligned}
$$

Since in the case of deterministic coefficients the value of $E\left[H_{\theta}(T)\right]$ is independent of $\theta \in \hat{\Theta}$, the above is equivalent to (noting that $\mu \geq 0$ )

$$
\begin{aligned}
& E\left[H_{\hat{\theta}}(T)\left(H_{\hat{\theta}}(T)-H_{\theta^{*}}(T)\right)\right]=0, \\
& \quad \text { and } E\left[H_{\hat{\theta}}(T)\left(H_{\theta}(T)-H_{\theta^{*}}(T)\right)\right] \geq 0 \quad \forall \theta \in \hat{\Theta} .
\end{aligned}
$$


Now,

$$
\begin{aligned}
H_{\hat{\theta}}(T) H_{\theta^{*}}(T) & \\
= & \exp \left\{-\int_{0}^{T}\left[2 r(t)+\frac{1}{2}\left(|\hat{\theta}(t)|^{2}+\left|\theta^{*}(t)\right|^{2}\right)\right] d t-\int_{0}^{T}\left[\hat{\theta}(t)+\theta^{*}(t)\right]^{\prime} d W(t)\right\} \\
= & \exp \left\{-\int_{0}^{T}\left[2 r(t)-\hat{\theta}(t)^{\prime} \theta^{*}(t)\right] d t\right\} \\
& \exp \left\{-\int_{0}^{T} \frac{1}{2}\left|\theta(t)+\theta^{*}(t)\right|^{2} d t-\int_{0}^{T}\left[\theta(t)+\theta^{*}(t)\right]^{\prime} d W(t)\right\} \\
= & \exp \left\{-\int_{0}^{T}\left[2 r(t)-|\hat{\theta}(t)|^{2}\right] d t\right\} \\
& \exp \left\{-\int_{0}^{T} \frac{1}{2}\left|\theta(t)+\theta^{*}(t)\right|^{2} d t-\int_{0}^{T}\left[\theta(t)+\theta^{*}(t)\right]^{\prime} d W(t)\right\},
\end{aligned}
$$

where we have used the identity $\hat{\theta}(t)^{\prime} \theta^{*}(t)=|\hat{\theta}(t)|^{2}$; see Lemma $7-(\mathrm{v})$. Thus, $E\left[H_{\hat{\theta}}(T) H_{\theta^{*}}(T)\right]=\exp \left\{-\int_{0}^{T}\left[2 r(t)-|\hat{\theta}(t)|^{2}\right] d t\right\}=E\left[H_{\hat{\theta}}(T)^{2}\right]$, which proves the first equality of (39). Next, thanks to the inequality in Lemma 7-(v), a similar calculation as above shows that, for any $\theta \in \hat{\Theta}, E\left[H_{\hat{\theta}}(T) H_{\theta}(T)\right]=$ $\exp \left\{-\int_{0}^{T}\left[2 r(t)-\hat{\theta}(t)^{\prime} \theta(t)\right] d t\right\} \geq \exp \left\{-\int_{0}^{T}\left[2 r(t)-|\hat{\theta}(t)|^{2}\right] d t\right\}=E\left[H_{\hat{\theta}}(T)^{2}\right]$. This, together with the proved first equality of (39), leads to the second inequality of (39).

Theorem 25. If $r(\cdot), \mu(\cdot)$ and $\sigma(\cdot)$ are deterministic, and $\sum_{j=1}^{m} \int_{0}^{T} B(t)_{j}^{+} d t>$ 0 where $B(t)_{j}$ denotes the $j$-th component of $B(t)$, then $\pi(t):=\left[\lambda e^{-\int_{t}^{T} r(s) d s}\right.$ $x(t)] v(t)$ is an efficient portfolio for the mean-variance problem (12) corresponding to $z \geq x_{0} e^{\int_{0}^{T} r(t) d t}$, where

$$
\lambda=\frac{z e^{\int_{0}^{T}|\hat{\theta}(t)|^{2} d t}-x_{0} e^{\int_{0}^{T} r(t) d t}}{e^{\int_{0}^{T}|\hat{\theta}(t)|^{2} d t}-1}, \quad \mu=\frac{z e^{\int_{0}^{T} r(t) d t}-x_{0} e^{\int_{0}^{T} 2 r(t) d t}}{e^{\int_{0}^{T}|\hat{\theta}(t)|^{2} d t}-1},
$$

and $v(\cdot)$ is an $\mathbf{R}_{+}^{m}$-valued measurable function satisfying $\sigma(t)^{\prime} v(t)=\hat{\theta}(t)$. Moreover, the efficient frontier is

$$
\operatorname{Var}(x(T))=\frac{1}{e^{\int_{0}^{T}|\hat{\theta}(t)|^{2} d t}-1}\left[z-x_{0} e^{\int_{0}^{T} r(t) d t}\right]^{2}, \quad z \geq x_{0} e^{\int_{0}^{T} r(t) d t} .
$$

Proof. By virtue of Lemma 24 and Theorem 23, $X^{*}:=\lambda-\mu H_{\hat{\theta}}(T)$ is the unique optimal solution to (35), provided that $\mu \geq 0$. The system of equations (16) reduces to

$$
\left\{\begin{array}{l}
\lambda-\mu E H_{\hat{\theta}}(T)=z \\
\lambda E H_{\hat{\theta}}(T)-\mu E\left[H_{\hat{\theta}}(T)^{2}\right]=x_{0},
\end{array}\right.
$$

where we have used the fact that $E\left[H_{\hat{\theta}}(T) H_{\theta^{*}}(T)\right]=E\left[H_{\hat{\theta}}(T)^{2}\right]$ which was proved in the proof of Lemma 24. When $\sum_{j=1}^{m} \int_{0}^{T} B(t)_{j}^{+} d t>0$, it must hold 
that $\int_{0}^{T}|\hat{\theta}(t)|^{2} d t>0$; therefore (40) is well-defined which gives the (only) solution pair to the above system, with $\mu \geq 0$ under the assumption that $z \geq x_{0} e^{\int_{0}^{T} r(t) d t}$.

Going through exactly the same argument that leads to (26), we have that an admissible portfolio $\pi(\cdot)$ is efficient if and only if it satisfies the no-shorting constraint and

$$
\sigma(t)^{\prime} \pi(t)=\mu e^{-\int_{t}^{T}\left(2 r(s)-|\hat{\theta}(s)|^{2}\right) d s} H_{\hat{\theta}}(t) \hat{\theta}(t) .
$$

By Lemma 7-(iii), there exists an $\mathbf{R}_{+}^{m}$-valued, $\mathcal{F}_{t}$-progressively measurable stochastic process $v(\cdot)$ satisfying $\sigma(t)^{\prime} v(t)=\hat{\theta}(t)$. Hence, the following portfolio

$$
\pi(t):=\mu e^{-\int_{t}^{T}\left(2 r(s)-|\hat{\theta}(s)|^{2}\right) d s} H_{\hat{\theta}}(t) v(t) \equiv\left[\lambda e^{-\int_{t}^{T} r(s) d s}-x(t)\right] v(t)
$$

indeed satisfies the no-shorting constraint as well as (42), and hence is efficient. The rest of the proof, in proving the form of the efficient frontier, is exactly the same as that of Theorem 18 .

\section{Concluding Remarks}

In this paper we have studied the mean-variance portfolio selection in a continuous-time incomplete market, with a no-shorting constraint on portfolios. One of the main results is that we have completely characterized, via some equivalent conditions, those contingent claims that are replicable by portfolios satisfying the constraint. This result per se is independent of the portfolio selection problem, and is more in the realm of risk hedging or option pricing. Nonetheless, this result has played a central role in handling the incompleteness of the market. Using a backward approach, we transferred the original mean-variance problems into static optimization problems on the terminal wealth, where all the original constraints including the budget constraint and incompleteness of market are translated into some terminal constraints. Solving these static constrained optimization problems using primal-dual convex optimization in Hilbert spaces has led to solution schemes for the underlying Markowitz problems and, in the case of deterministic opportunity set, to complete and closed-form solutions.

While the continuous-time portfolio selection models with the security price processes governed by geometric Brownian motions are considered in this paper, we believe that our results extend to semimartingale models, including the discrete-time case, with necessary technical modifications, some of which may be straightforward and some may be involved. However, we chose to use the current setup, as what we have hitherto done in our related works, for the main reason that we do not want to let unduly technicality blur the financial essence of the results and distract the reader's attention. 


\section{Appendix}

\section{A Some lemmas}

We present several technical lemmas that are useful in the main context. We start with the following result which is originally due to Frank and Wolfe [9]. A complete proof (for a mode general case) can be found in [21].

Lemma 26. If a quadratic function $f: \mathbf{R}^{d} \rightarrow \mathbf{R}$ is bounded below on a nonempty polyhedron $S$, then $f$ attains its infimum on $S$.

Lemma 27. Given $a \in \mathbf{R}^{n}$ and $A \in \mathbf{R}^{m \times n}$. If $a \notin\left\{A^{\prime} u: u \in \mathbf{R}_{+}^{m}\right\}$, then there exists $v \in \mathbf{R}^{n} \backslash\{0\}$ such that $a^{\prime} v=-1$ and $A v \geq 0$.

Proof. By the assumption $a \neq 0$. Denote $M:=\left\{w \in \mathbf{R}^{n}: a^{\prime} w<0\right\}, N:=$ $\left\{w \in \mathbf{R}^{n}: A w \geq 0\right\}$, which are both nonempty convex cones. If $M \cap N=$ $\emptyset$, then by the convex separation theorem, there exists $y \in \mathbf{R}^{n} \backslash\{0\}$ with $\sup _{w \in A} y^{\prime} w \leq \inf _{w \in B} y^{\prime} w$. This implies

$$
y^{\prime} w \leq 0 \forall w \text { with } a^{\prime} w<0
$$

and

$$
y^{\prime} w \geq 0 \forall w \text { with } A w \geq 0 .
$$

It follows from (43) that there exists $k>0$ such that $a=k y$. On the other hand, (44) together with Farkas' lemma (see, e.g., [1, p.58, Theorem 2.9.1]) yields there is $\pi \in \mathbf{R}_{+}^{m}$ such that $y=A^{\prime} \pi$. So $a=k y=A^{\prime}(k \pi) \in\left\{A^{\prime} u: u \in\right.$ $\left.\mathbf{R}_{+}^{m}\right\}$, leading to a contradiction. Hence $M \cap N \neq \emptyset$. The desired conclusion then follows immediately.

Before we state the next lemma, we note that a set $A \subset[0, T] \times \Omega$ is said to be $\mathcal{F}_{t}$-progressive is the corresponding indicator function $1_{A}$ is $\mathcal{F}_{t^{-}}$ progressively measurable. The $\mathcal{F}_{t}$-progressive sets form a $\sigma$-field (see, e.g., $[13$, p.99]).

Lemma 28. Let $X \equiv\{X(t): 0 \leq t \leq T\}$ be a given $n$-dimensional, $\mathcal{F}_{t}$ progressively measurable stochastic process. Assume that $S(t, \omega):=\left\{y \in \mathbf{R}^{m}\right.$ : $f(X(t, \omega), y) \leq 0\} \neq \varnothing$ for any $(t, \omega) \in[0, T] \times \Omega$, where $f: \mathbf{R}^{n} \times \mathbf{R}^{m} \rightarrow \mathbf{R}^{k}$ is jointly measurable in both variables and continuous in the second variable. Then the process $\alpha \equiv\{\alpha(t): 0 \leq t \leq T\}$ defined as $\alpha(t, \omega):=$ $\operatorname{argmin}_{y \in S(t, \omega)}|y|^{2}$ is also $\mathcal{F}_{t}$-progressively measurable.

Proof. First of all, for each $(t, \omega) \in[0, T] \times \Omega, S(t, w)$ is a closed set, and the square function is strictly convex and coercive. Hence $\alpha(t, \omega)$ is well defined. Set $g(t, \omega):=|\alpha(t, \omega)|^{2}$. Then for any $x \in \mathbf{R}$,

$$
\{(t, \omega): g(t, \omega)<x\}=\cup_{v \in \mathbf{Q}^{m},|v|^{2}<x}\{(t, \omega): f(X(t, \omega), v) \leq 0\} .
$$


This shows that $g$ is $\mathcal{F}_{t}$-progressively measurable.

Denote $S_{n}(t, \omega):=S(t, \omega) \cap\left\{y \in \mathbf{R}^{m}:|y|^{2} \leq g(t, \omega)+1 / n\right\}$, for $(t, \omega) \in$ $[0, T] \times \Omega$, and $n=1,2, \cdots$. Fix $n$. For any open set $O \subset \mathbf{R}^{m}$, we have

$$
\begin{aligned}
& \left\{(t, \omega): S_{n}(t, \omega) \cap O \neq \varnothing\right\}= \\
& \cup_{v \in O \cap \mathbf{Q}^{m}}\left\{(t, \omega): f(X(t, \omega), v) \leq 0,|v|^{2} \leq g(t, \omega)+1 / n\right\},
\end{aligned}
$$

which is therefore an $\mathcal{F}_{t}$-progressive set. This shows that $S_{n}(t, \omega)$ satisfies the condition required in the measurable selection theorem [3, p. 281, Theorem 8.3.ii]. Hence, there exists an $\mathcal{F}_{t}$-progressively measurable process $\alpha_{n}$ with $\alpha_{n}(t, \omega) \in S_{n}(t, \omega)$ almost surely on $[0, T] \times \Omega$. It is clear that $\alpha_{n}(t, \omega) \rightarrow$ $\alpha(t, \omega)$, almost surely, as $n \rightarrow \infty$. Thus $\alpha$ is $\mathcal{F}_{t}$-progressively measurable.

\section{B Proof of Lemma 7}

(i) First of all, $\theta^{*}$ is clearly well-defined by (10). By Lemma $28, \theta^{*}$ is an $\mathcal{F}_{t}$-progressively measurable process. Moreover, due to Assumption (A) we must also have $\theta^{*} \in L_{\mathcal{F}}^{\infty}\left(0, T, \mathbf{R}^{n}\right)$. Similarly, one can prove $\hat{\theta} \in L_{\mathcal{F}}^{\infty}\left(0, T, \mathbf{R}^{n}\right)$ (indeed $|\hat{\theta}(t)| \leq\left|\theta^{*}(t)\right|$ by their definitions).

(ii) Pointwisely in $(t, \omega)$ (other than those points in a $(t, \omega)$-null set), $\theta^{*}(t)$ minimizes $|\theta|^{2}$ subject to $\sigma(t) \theta=B(t)$. Hence by the Lagrange approach there is $u \in \mathbf{R}^{m}$ so that $\theta^{*}(t)$ minimizes $|\theta|^{2}-2[\sigma(t) \theta-B(t)]^{\prime} u$ over $\theta \in \mathbf{R}^{n}$. The zero-derivative condition then gives $\theta^{*}(t)=\sigma(t)^{\prime} u$. This implies that $\{u \in$ $\left.\mathbf{R}^{m}: \sigma(t)^{\prime} u=\theta^{*}(t)\right\} \neq \emptyset$. Define $u(t):=\operatorname{argmin}_{u \in\left\{u \in \mathbf{R}^{m}: \sigma(t)^{\prime} u=\theta^{*}(t)\right\}}|u|^{2}$. Then by virtue of Lemma $28 u(\cdot)$ is the desired process.

(iii) For each fixed $(t, \omega)$ not in a $(t, \omega)$-null set, $\hat{\theta}(t)$ minimizes $|\theta|^{2}$ subject to $\sigma(t) \theta \geq B(t)$. By the Kuhn-Tucker theorem there exists $v \in \mathbf{R}_{+}^{m}$ such that $\hat{\theta}(t)$ minimizes $|\theta|^{2}-2[\sigma(t) \theta-B(t)]^{\prime} v$ over $\theta \in \mathbf{R}^{n}$. This leads to $\hat{\theta}(t)=\sigma(t)^{\prime} v$. The rest of the proof is the same as in (ii) above.

(iv) For any $\theta \in \Theta$, we have, by (ii), that $\theta^{*}(t)^{\prime} \theta(t)=u(t)^{\prime} \sigma(t) \theta(t)=$ $u(t)^{\prime} B(t)=u(t)^{\prime} \sigma(t) \theta^{*}(t)=\left|\theta^{*}(t)\right|^{2}$.

(v) We continue with the argument in proving (iii) above. It follows from the Kuhn-Tucker theorem that there exists $v \in \mathbf{R}_{+}^{m}$ such that $\hat{\theta}(t)=\sigma(t)^{\prime} v$ and $v^{\prime}[\sigma(t) \hat{\theta}(t)-B(t)]=0$. So for any $\theta(\cdot) \in \hat{\Theta}$,

$$
\hat{\theta}(t)^{\prime} \theta(t)=v^{\prime} \sigma(t) \theta(t) \geq v^{\prime} B(t)=v^{\prime} \sigma(t) \hat{\theta}(t)=|\hat{\theta}(t)|^{2} .
$$

Moreover, the only inequality in the above becomes equality when $\theta(\cdot)=\theta^{*}(\cdot)$. This proves the desired results.

\section{References}

1. A.V. Balakrishnan, Applied Functional Analysis, Springer-Verlag, New York, 1976. 
2. T.R. Bielecki, H. Jin, S.R. Pliska And X.Y. Zhou, Continuous-time meanvariance portfolio selection with bankruptcy prohibition, Math. Finance, 15 (2005), pp. 213-244.

3. L. Cesari, Optimization-Theory and Applications, Springer-Verlag, New York, 1985

4. S. Chen, X. Li AND X. Zhou, Stochastic linear quadratic regulators with indefinite control weight costs, SIAM J. Contr. Optim., 36 (1998), pp. 1685-1702.

5. J. CVitanic And I. Karatzas, Convex duality in constrained portfolio optimization, Ann. Appl. Prob., 2 (1992), pp. 767-818.

6. J. Cvitanic And I. Karatzas, Hedging contingent claims with constrained portfolios, Ann. Appl. Probab., 3 (1993), pp. 652-681.

7. N. El Karoui, S. Peng And M.C. Quenez, Backward stochastic differential equations in finance, Math. Finance, 7 (1997), pp. 1-71.

8. N. El Karoui And M.C. Quenez, Dynamic programming and pricing of contingent claims in an incomplete market. SIAM J. Contr. Optim. 33 (1995), pp. 29-66.

9. M. FRAnK AND P. Wolfe, An algorithm for quadratic programming, Naval Res. Logistics Quart., 3 (1956), pp. 95-110.

10. H. He AND N. PeARson, Consumption and portfolio policies with incomplete markets and short-sale constraints: The finite-dimensional case, Math. Finance, 1 (1991), pp. 1-10.

11. H. He AND N. Pearson, Consumption and portfolio policies with incomplete markets: The infinite-dimensional case, J. Econom. Theory, 54 (1991), 259-305.

12. J.M. HARRISON AND D. KREPS, Martingales and multiperiod securities market, J. Econom. Theory, 20 (1979), pp. 381-408.

13. O. Kallenberg, Foundations of Modern Probability, Springer, Berlin, 1997.

14. I. Karatzas, J.P. Lehoczky, S.E. Shreve And G. Xu, Martingale and duality methods for utility maximization in an incomplete market, SIAM J. Control Optim., 29 (1991), pp. 702-730.

15. I. Karatzas And S.E. Shreve, Methods of Mathematical Finance, SpringerVerlag, New York, 1998.

16. D. Kramkov, Optional decomposition of supermartingales and hedging contingent claims in incomplete security markets, Probab. Theory Rel. Fields 105 (1996), pp. 459-479.

17. D. LI AND W.L. NG, Optimal dynamic portfolio selection: Multiperiod meanvariance formulation, Math. Finance, 10 (2000), pp. 387-406.

18. X. LI, X.Y. Zhou AND A.E.B. Lim, Dynamic mean-variance portfolio selection with no-shorting constraints, SIAM J. Control Optim., 40 (2001), pp. 1540-1555.

19. A.E.B. LiM, Quadratic hedging and mean-variance portfolio selection with random parameters in an incomplete market, Math. Oper. Res., 29 (2004), pp. 132-161.

20. A.E.B. Lim AND X.Y. ZHOU, Mean-variance portfolio selection with random parameters, Math. Oper. Res., 27 (2002), pp. 101-120.

21. Z.Q. LOU AND S. Zhang, On extensions of the Frank-Wolfe theorems, Comput. Optim. Appl., 13 (1999), pp. 87-110.

22. D.G. Luenberger, Optimization by Vector Space Methods, Wiley, New York, 1969.

23. H. Markowitz, Portfolio selection, J. of Finance, 7 (1952), pp. 77-91.

24. H. Markowitz, Private communication, 2004. 
25. J. von Neumann and O. Morgenstern, Theory of Games and Economic Behavior, 2nd Edition, Princeton University Press, Princeton, New Jersey, 1947.

26. S.R. PliskA, A discrete time stochastic decision model, Advances in Filtering and Optimal Stochastic Control, edited by W.H. Fleming and L.G. Gorostiza, Lecture Notes in Control and Information Sciences, 42, Springer-Verlag, New York, 290-304, 1982.

27. W. Schachermayer, Optimal investment in incomplete markets when wealth may become negative, Ann. Appl. Probab., 11 (2001), pp. 694-734.

28. M.C. SteinbaCH, Markowitz revisited: Mean-variance models in financial portfolio analysis, SIAM Rev., 43 (2001), pp. 31-85.

29. J. Yong And X.Y. Zhou, Stochastic Controls: Hamiltonian Systems and HJB Equations, Springer, New York, 1999.

30. X.Y. Zhou AND D. LI, Continuous time mean-variance portfolio selection: A stochastic LQ framework, Appl. Math. Optim., 42 (2000), pp. 19-33. 